\title{
Monodispersed DOTA-PEG-Conjugated Anti-TAG-72 Diabody Has Low Kidney Uptake and High Tumor-to-Blood Ratios Resulting in Improved ${ }^{64} \mathrm{Cu}$ PET
}

Lin $\mathrm{Li}^{* 1}$, Fabio Turatti*2, Desiree Crow*3, James R. Bading ${ }^{3}$, Anne-Line Anderson ${ }^{3}$, Erasmus Poku ${ }^{3}$, Paul J. Yazaki ${ }^{3}$, Lawrence E. Williams ${ }^{4}$, Debra Tamvakis ${ }^{2}$, Paul Sanders ${ }^{2}$, David Leong ${ }^{2}$, Andrew Raubitschek ${ }^{3}$, Peter J. Hudson ${ }^{\dagger 2}$, David Colcher ${ }^{\dagger 3}$, and John E. Shively ${ }^{\dagger 1}$

${ }^{I}$ Department of Immunology, Beckman Research Institute of City of Hope, Duarte, California; ${ }^{2}$ Avipep Pty. Ltd., Parkville, Victoria, Australia; ${ }^{3}$ Department of Cancer Immunotherapy and Tumor Immunology, Beckman Research Institute of City of Hope, Duarte, California; and ${ }^{4}$ Department of Diagnostic Radiology, City of Hope, Duarte, California

Diabodies are noncovalent dimers of single-chain antibody fragments that retain the avidity of intact IgG but have more favorable blood clearance than intact lgG. Radiometals offer a wide range of half-lives and emissions for matching imaging and therapy requirements and provide facile labeling of chelate-antibody conjugates. However, because of their high retention and metabolism in the kidney, the use of radiometal-labeled diabodies can be problematic for both imaging and therapy. Methods: Having previously shown that ${ }^{111}$ In-DOTA-polyethylene glycol (PEG)3400-anti-carcinoembryonic antigen diabody has less than half the kidney uptake and retention of non-PEGylated diabody and that the two have similarly high tumor uptake and retention, we synthesized a similar derivative for an antitumor-associated glycoprotein 72 diabody. We also reduced the molecular size of the polydispersed PEG3400 to monodispersed PEG27 and PEG12 (nominal masses of 1,321 and 617, respectively). We performed biodistributions of their DOTA conjugates radiolabeled with ${ }^{125} \mathrm{I},{ }^{111} \mathrm{In}$, or ${ }^{64} \mathrm{Cu}$ in tumor-bearing athymic mice. Results: The addition of PEG3400 to the diabody reduced kidney uptake to a level $(\approx 10$ percentage injected dose/ g) comparable to that obtained with radiometal-labeled intact IgG. The PEG27 and PEG12 diabody conjugates also demonstrated low kidney uptake without reduction of tumor uptake or tumor-to-blood ratios. When radiolabeled with ${ }^{64} \mathrm{Cu}$, the DOTA-PEG12 and -PEG27 diabody conjugates gave highcontrast PET images of colon cancer xenografts in athymic mice. Conclusion: PEGylated diabodies may be a valuable platform for delivery of radionuclides and other agents to tumors.

Key Words: diabody; CC49; TAG-72; DOTA; ${ }^{64} \mathrm{Cu}$, polyethylene glycol; PET

J Nucl Med 2010; 51:1139-1146

DOI: 10.2967/jnumed.109.074153

Received Dec. 20, 2009; revision accepted Mar. 17, 2010.

For correspondence or reprints contact: John E. Shively, City of Hope,

1450 E. Duarte Rd., Duarte, CA 91010.

E-mail: jshively@coh.org

${ }^{*}$ Contributed equally to this work.

${ }^{\dagger}$ Contributed equally to this work.

COPYRIGHT @ 2010 by the Society of Nuclear Medicine, Inc.
$\mathbf{T}$ o be effective tumor imaging agents, targeted radiolabeled antibodies must provide both high tumor uptake and high tumor-to-blood ratios $(1,2)$. At one extreme, monoclonal antibodies $(150 \mathrm{kDa})$ have a relatively slow blood clearance $\left(\beta\right.$-half-life $\left[\mathrm{t}_{1 / 2 \beta}\right], 48-72 \mathrm{~h}$ ), allowing ample time for accumulation in the tumor, but suffer from low tumor-toblood ratios. At the other extreme, single-chain $\mathrm{Fv}$ ( $\mathrm{scFv}$ ) recombinant fragments $(25 \mathrm{kDa})$ are rapidly cleared from the blood $\left(\mathrm{t}_{1 / 2 \beta}, 0.5-2.0 \mathrm{~h}\right)$, resulting in high tumor-to-blood ratios but low overall accumulation of radioactivity in tumor (3). After analyzing a series of radioiodinated recombinant antibody fragments (4), we and others concluded that simultaneous achievement of high tumor uptake and high tumor-to-blood ratio requires a bivalent antibody of size 50$80 \mathrm{kD}(1,3,4)$, with diabodies (noncovalent dimers of size 55 $\mathrm{kDa}$ ) as one platform fulfilling these criteria. For example, ${ }^{124}$ I-labeled diabodies have performed well in a preclinical model of PET (5). However, a drawback to radioiodinated antibodies is that they may be rapidly metabolized in tissues, including tumor, especially if the antigen undergoes internalization on antibody binding. Radiometal-labeled recombinant antibody fragments offer a significant improvement in tumor-to-blood ratio, but this is offset by high kidney retention. Thus, systemic excretion of radiometal metabolites is slow, compared with radioiodine metabolic products. We have identified ${ }^{111}$ In-DOTA- $\varepsilon$-lysine as the metabolic endpoint product in the kidney for ${ }^{111}$ In-DOTA anticarcinoembryonic antigen (CEA) antibody fragments (6).

We and others have sought to improve the utility of radiometal-labeled recombinant antibody fragments by decreasing kidney uptake or retention. In one approach, kidney uptake is partially blocked by the administration of intravenous L- or D-lysine $(7,8)$. Alternatively, we have designed unique metabolizable linkers between the antibody 
and DOTA that have decreased kidney retention up to 4-fold compared with $N$-hydroxy succinimide (NHS)DOTA conjugates (9). A third approach is to decrease kidney retention of antibody fragments by increasing their apparent molecular weight by attachment of polyethylene glycol (PEG) (10). This approach was successfully applied to anti-CEA $\mathrm{F}\left(\mathrm{ab}^{\prime}\right)$ fragment $\mathrm{A} 5 \mathrm{~B} 7$ by conjugating it to MPEG6000, which increased tumor retention and serum half-life by 6- and 5-fold, respectively (11). In the case of antimucin scFv CC49/218 conjugated to PEG5000, a 14-fold increase in serum half-life was observed (12). In that study, the authors concluded that greater improvements were observed with increasing size of PEG polymers rather than total amount of PEG, which often led to decreased immunoreactivity of the conjugate. In another study, investigators engineered an anti-mucin-1 dimeric scFv-thiol (55 kDa) with C-terminal cysteine residues for site-specific conjugation to maleimide-PEGs of different sizes (13). They concluded that conjugation yields needed improvement before clinical application. Interestingly, a recent report (14) evaluated a $40-\mathrm{kDa}$ branched-chain PEG conjugated to a bispecific diabody that bound covalently to CEA and CD3 and showed that PEGylation doubled tumor uptake and increased circulation time $\left(\mathrm{t}_{1 / 2 \beta}, 50 \mathrm{~h}\right)$. Along the same line of investigation, Leong et al. (15) used the natural thiol group of an engineered $\mathrm{Fab}^{\prime}$ fragment to IL- 8 for conjugation to maleimide-PEGs of different sizes. They found that conjugation to maleimide PEG $10 \mathrm{kDa}$ resulted in an apparent molecular size of $180 \mathrm{kDa}$, comparable to the size of an intact antibody. Importantly, blood clearance studies revealed a $t_{1 / 2 \beta}$ of $44 \mathrm{~h}$, compared with $3 \mathrm{~h}$ for the unmodified Fab'. Also, Yang et al. (10) conjugated maleimide PEGs of different sizes to an anti-tumor necrosis factor- $\alpha$ scFv (half the size of a diabody) with a cysteine engineered into the linker region or $\mathrm{C}$ terminus. They found that conjugation to maleimide PEG (20 kDa) gave a blood clearance half-life similar to that of intact IgG but offered no conclusion regarding the effect of the PEG modification on kidney retention.

Recently, we have shown that adding PEG3400 to ${ }^{111} \mathrm{In}-$ DOTA-anti-CEA diabody reduced kidney uptake at 24-48 h from over 200 percentage injected dose (\%ID)/g for the nonPEGylated diabody to $50 \% \mathrm{ID} / \mathrm{g}$ while preserving high tumor uptake (16). However, further reduction in kidney uptake is required. To study diabody dependence of DOTAPEG3400 conjugation, we generated and PEGylated an antitumor-associated glycoprotein 72 (TAG-72) diabody for which kidney retention was reduced to levels $(10 \% \mathrm{ID} / \mathrm{g}$ at 1-24 h) usually seen with intact IgG. This suggested that kidney uptake is a function of both apparent molecular size (as modified by PEG) and other less-defined factors intrinsic to the diabody (e.g., modification of isoelectric point [pI] has been shown to influence kidney uptake $(17,18))$.

Having reduced the kidney uptake for radiometal-labeled diabody by conjugation to PEG3400, we sought to further investigate the approach by using monodispersed DOTAPEG conjugates. We synthesized DOTA-PEG27-Cys-VS and DOTA-PEG12-Cys-VS, where Cys-VS is vinyl sulfone conjugated to the thiol of cysteine, and conjugated these monodispersed PEGs to amino groups on the diabody at $\mathrm{pH}$ 9.0, a reaction we have previous described (19). The conjugates were characterized by mass spectrometry, isoelectric focusing (IEF), and size-exclusion chromatography. Biodistributions of ${ }^{111}$ In-labeled conjugates in athymic mice bearing LS-174T colon tumor xenografts gave tumor uptakes of 40-45 \% ID/g, tumor-to-blood ratios of 8-10, and a kidney uptake of $10 \%$ ID/g at $24 \mathrm{~h}$. Furthermore, PET with ${ }^{64} \mathrm{Cu}$-labeled conjugates provided high-contrast tumor images within $24 \mathrm{~h}$ after injection (tumor-to-blood ratio, $8: 1)$.

\section{MATERIALS AND METHODS}

\section{Materials, Radiolabels, Mass Spectrometry}

LS-174T cells were obtained from ATCC and maintained as previously described (16). 1,4,7,10-Tetraazacyclododecane-1,4,7$\operatorname{tris}(t$-butyl acetate)-10-acetic acid was obtained from Macrocyclics, Inc. NHS-PEG3400-VS (catalog no. 4M5B0F02) was purchased from Nektar. $N$-fluorenylmethyloxycarbonyl (FMOC)amido-dPEG27 acid was obtained from Novabiochem (EMD Biosciences), and $\mathrm{N}$-FMOC-amido-dPEG12 acid was purchased from Quanta Biodesign Ltd. ${ }^{111} \mathrm{In}$-chloride was from Amersham, ${ }^{64} \mathrm{Cu}$ from Washington University School of Medicine, and ${ }^{125} \mathrm{I}$ from Perkin Elmer. Mass spectra were recorded on an Agilent 6520 quadrupole time-of-flight liquid chromatography mass spectrometry device.

\section{Construction, Cloning, Expression, and Purification of Anti-TAG-72 Diabody}

The anti-TAG72 diabody (AVP04-07) was based on V-domains from the parent $\mathrm{CC} 49$ antibody and derivative $\mathrm{scFv}$ fragments. The gene had codons optimized for Escherichia coli expression with the orientation $\mathrm{V}_{\mathrm{H}}-\mathrm{V}_{\mathrm{L}}$ (where $\mathrm{V}_{\mathrm{H}}$ and $\mathrm{V}_{\mathrm{L}}$ are the variable domains of the heavy and light chains, respectively) joined by a $\mathrm{G}_{4} \mathrm{~S}$ linker and included a C-terminal $\mathrm{His}_{6}$ tail. AVP04-07 was subcloned into $E$. coli $\mathrm{BL} 21(\mathrm{DE} 3)\left(\mathrm{F}^{-}\right.$ompT hsd $S_{\mathrm{B}}\left(\mathrm{r}_{\mathrm{B}}{ }^{-} \mathrm{m}_{\mathrm{B}}{ }^{-}\right)$gal $\mathrm{dcm}$ (DE3)) (Novagen) and produced in an $18 \mathrm{~L}$ fermenter. Bacterial lysate cleared by centrifugation $(16,000 \times g, 30 \mathrm{~min})$ and filtration at $0.45 \mu \mathrm{m}$ was purified using a 3-step procedure (HisTrap, HiTrap SP HP, and Superdex 75 prep chromatography; GE Healthcare) on an AKTA Purifier 10 (GE Healthcare).

\section{Analytic Ultracentrifugation}

Sedimentation velocity experiments were conducted using a Beckman model XL-I analytic ultracentrifuge at $20^{\circ} \mathrm{C}$. Data were collected at $\mathrm{A}_{290 \mathrm{~nm}}$ in continuous mode, using a time interval of $300 \mathrm{~s}$ and a step size of $0.003 \mathrm{~cm}$, without averaging. Solvent density $\left(1.00499 \mathrm{~g} / \mathrm{mL}\right.$ at $\left.20^{\circ} \mathrm{C}\right)$, solvent viscosity $(1.0214$ $\mathrm{cP}$ ), and partial specific volume for AVP04-07 were computed using the program SEDNTERP. Sedimentation velocity data were fitted to a continuous size-distribution model using the program SEDFIT (www.analyticalultracentrifugation.com).

\section{Immunoreactivity and IEF}

Binding of AVP04-07 to the TAG-72 antigen was determined using bovine submaxillary mucin (Aldrich-Sigma) in a column 
shift assay (Superdex 200). IEF gel electrophoresis was run either on an IEF Precast gel (pH 3-10) (Novex) or on a Pharmacia PhastGel according to the manufacturer's instructions.

\section{Conjugation of AVP04-07}

NHS-DOTA was conjugated to AVP04-07 diabody as previously described (16). NHS-PEG3400-VS was conjugated to AVP04-07 diabody at a molar ratio of 30:1 and $\mathrm{pH} 6.0$ as previously described (19). The conjugate was reacted with cysteine amido-DOTA (20) at a molar ratio of 50:1. $N$-FMOCamido-PEG27 acid was coupled to Cys-polystyrene Wang resin, cleaved and purified by reverse-phase high-performance liquid chromatography, reacted with a 10-molar excess of vinyl sulfone, and repurified by reverse-phase high-performance liquid chromatography. DOTA-PEG27-Cys-VS was reacted with AVP04-07 diabody at a molar ratio of 50:1. Synthesis and conjugation of DOTA-PEG12-Cys-VS to diabody at molar ratios of 20:1 and 50:1 were performed as described above.

\section{Radiolabeling of AVP04-07 and Its Conjugates}

AVP04-07 and its conjugates was radioiodinated with ${ }^{125} \mathrm{I}$ (Perkin Elmer) using the IODO-GEN method (21). Na ${ }^{125}$ I (5$10 \mu \mathrm{L}, 26 \mathrm{mBq}$ ) was added to $200 \mu \mathrm{g}$ of AVP04-07 in a tube precoated with $20 \mu \mathrm{g}$ of IODO-GEN (Pierce) and incubated at room temperature for $3 \mathrm{~min}$. Radiolabeling yields were $80 \%-100 \%$. Radiometal labeling of DOTA-AVP04-07 was performed using ${ }^{111} \mathrm{InCl}_{3}(0.15 \mathrm{mBq} / \mu \mathrm{g})$ or ${ }^{64} \mathrm{CuCl}_{2}(0.37 \mathrm{mBq} / \mu \mathrm{g})$ as previously described (19). Radiolabeling yields were typically $70 \%-90 \%$. The radiolabeled material was purified on Superdex75 or -200 columns.

\section{LS-174T Xenograft Model}

Female, athymic nu/nu mice (Charles River Laboratories), 6-8 wk old, were injected with LS-174T cells $\left(10^{6}\right)$ subcutaneously in the flank. After $10 \mathrm{~d}$, mice were injected intravenously with a mixture $(200 \mu \mathrm{L})$ of $370 \mathrm{kBq}$ of ${ }^{125}$ I-labeled and $150 \mathrm{kBq}$ of ${ }^{111}$ In-labeled AVP04-07 (2-6 $\mu \mathrm{g}$ of total protein) for biodistribution studies. Mice were euthanized at various time points: tumor, blood, and major organs were collected, weighed, and counted with correction for crossover of ${ }^{111}$ In counts into the ${ }^{125} \mathrm{I}$ channel. Time-activity curves were corrected for radioactive decay and presented as $\% \mathrm{ID} / \mathrm{g}$.

\section{PET}

Tumor-bearing mice were injected intravenously with ${ }^{64} \mathrm{Cu}$ labeled diabody or diabody-PEG conjugates and imaged at 1, 4, 21-22, and 45-46 h with a small-animal PET scanner (microPET model R4; Siemens/CTIMI). Mice anesthetized with isoflurane were scanned for $20 \mathrm{~min}$ at the 1- and 4-h time points, $45 \mathrm{~min}$ at $21-22 \mathrm{~h}$, and $60 \mathrm{~min}$ at $45-46 \mathrm{~h}$. Data were sorted into 2-dimensional sinograms using the Fourier rebinning method and corrected for intrascan radiodecay, detector nonuniformity, and random coincidence noise. Images were reconstructed by the iterative ordered-subsets expectation maximization method (4 iterations, 16 subsets).

\section{Comparison of Imaging Characteristics Among Diabody} and Derivatives

An imaging figure of merit (IFOM) was applied to both planar and PET images $(1,2,16)$. The IFOM at time $t$ after injection is defined as:

$$
\begin{aligned}
\operatorname{IFOM}(t) & =(-1)^{a} \mathrm{CV}_{0}(z)^{2} \varepsilon u_{T} \exp (-\lambda t) A_{0} \operatorname{Vol}[1-1 / r]^{2} /[1+1 / r] \\
r & =u_{T} / u_{B} ; a=0 \quad \text { when } \quad r \geq 1, a=1 \quad \text { when } \quad r<1,
\end{aligned}
$$

with $\mathrm{CV}_{0}$ being the coefficient of variation of the difference in tumor and background counts $(z), \varepsilon$ the detector efficiency factor, $\lambda$ the decay constant, $A_{0}$, the total injected activity and Vol the tumor volume. Uptake (\%ID/g) was denoted as $u_{T}$ (tumor) and $u_{B}$ (background), respectively. In plotting this function, we set the product of $\mathrm{CV}_{0}, \varepsilon, A_{0}$, and $\mathrm{Vol}$ equal to unity. The only sources of variation are tumor uptake, background tissue uptake, and physical decay. Note that $\operatorname{IFOM}(t)<0$ implies that background tissue (blood or kidney) has greater uptake than tumor at time $t$.

\section{RESULTS}

\section{Characterization of AVP04-07 Diabody}

AVP04-07 diabody expressed in E. coli and purified by a 3-step method was eluted as a single species on gel filtration (Supplemental Fig. 1A; supplemental materials are available online only at http://jnm.snmjournals.org) as a monodispersed dimer with an apparent molecular mass of $52.5 \mathrm{kDa}$ by analytic ultracentrifugation (Supplemental Fig. 1B). It had more than $99 \%$ immunoreactivity as judged by a column shift assay (Supplemental Fig. 1C).

\section{Biodistribution of ${ }^{111} \|$ n-DOTA- and ${ }^{125}$-AVP04-07 in Athymic Mouse LS-174T Xenograft Model}

${ }^{125}$ I-DOTA-AVP04-07 and ${ }^{111}$ In-DOTA-AVP04-07 had similar blood clearances, with about $50 \%$ of the radiolabel removed by $1 \mathrm{~h}$ after injection and $10 \%$ in circulation at $4 \mathrm{~h}$ (Figs. 1A and 1B). Kidney uptake was high (100\% ID/g at $24 \mathrm{~h}$ ) for ${ }^{111}$ In-labeled but not ${ }^{125}$ I-labeled diabody, demonstrating that the kidney was the major route of clearance. For the ${ }^{111}$ In-labeled diabody there was significant tumor uptake, with over $25 \% \mathrm{ID} / \mathrm{g}$ observed as early as $4 \mathrm{~h}$ after injection and more than $20 \% \mathrm{ID} / \mathrm{g}$ retained at $48 \mathrm{~h}$. The tumor-to-blood ratio for ${ }^{111} \mathrm{In}-\mathrm{AVP04}-07$ was more than 50:1 at 24 h. ${ }^{125}$ I-labeled AVP04-07 gave tumor uptakes of 17 and $10 \% \mathrm{ID} / \mathrm{g}$ at 4 and $48 \mathrm{~h}$, respectively.

\section{Synthesis and Biodistribution of DOTA-Cys-VS- PEG3400-AVP04-07}

Since PEGylation reduces kidney uptake of radiometallabeled diabodies, we first conjugated AVP04-07 to DOTACys-VS-PEG3400-NHS as previously described for an anti-CEA diabody (16). VS-PEG3400-NHS was conjugated to surface lysines of the diabody (via active ester), followed by reaction with DOTA-Cys (20). Analysis by sodium dodecylsulfate and IEF gel electrophoresis indicate an expected shift to lower pI (IEF gel, Supplemental Fig. 2A, lane 5), as well as a shift to higher apparent molecular size due to the addition of PEG3400 (sodium dodecylsulfate gel, Supplemental Fig. 2B, lane 5). The conjugate was radiolabeled with ${ }^{111} \mathrm{InCl}_{3}$, chromatographed on Superdex 75, and compared with unconjugated AVP04-07 (Supplemental Figs. 2A and 2B). The apparent molecular weight of the PEG3400 derivative was $105 \mathrm{kDa}$, versus $52.5 \mathrm{kDa}$. The 


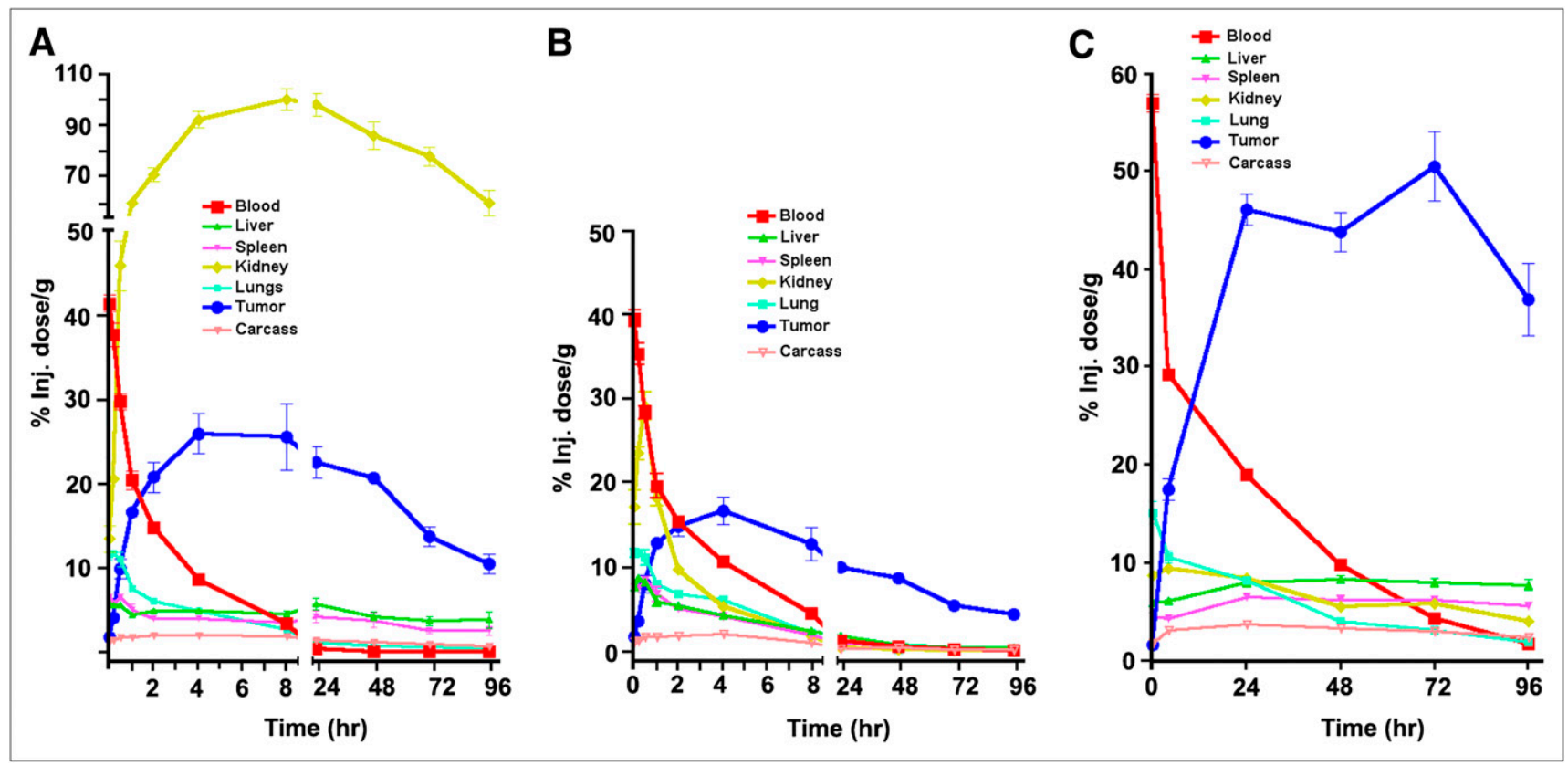

FIGURE 1. Biodistributions of unconjugated and DOTA-Cys-VS-PEG3400-conjugated AVP04-07 measured in athymic mice bearing LS-174T xenografts: ${ }^{111} \mathrm{In}$-labeled non-PEGylated diabody (A), ${ }^{125}$ I-labeled intact diabody (B), and ${ }^{111} \mathrm{In}-\mathrm{labeled}$ PEG3400 conjugated diabody (C).

increase can be attributed to the effect of PEGylation on the Stokes radius and is similar to the shift in size we observed for PEG3400-derivatized anti-CEA diabody (16).

The biodistribution of ${ }^{111}$ In-AVP04-07-PEG3400 is shown in Figure 1C. Kidney uptake was $98 \% \mathrm{ID} / \mathrm{g}$ at $24 \mathrm{~h}$ for non-PEGylated diabody (Fig. 1A) and $8.4 \% \mathrm{ID} / \mathrm{g}$ at $24 \mathrm{~h}$ for PEGylated diabody (Fig. 1C). The large reduction of kidney uptake with PEGylation was accompanied by an increase in tumor uptake from 22 to $46 \% \mathrm{ID} / \mathrm{g}$ at $24 \mathrm{~h}$ and a reduction in tumor-to-blood ratio ( $>46: 1$ to $2: 1$ at $24 \mathrm{~h}$ ). The increase in tumor retention is evidently due to the prolonged blood clearance of PEGylated diabody $\left(\mathrm{t}_{1 / 2 \beta}\right.$, $36 \mathrm{~h})$ versus non-PEGylated diabody $\left(\mathrm{t}_{1 / 2 \beta}, 18 \mathrm{~h}\right)$. The reduction in kidney uptake was much greater than previously observed with ${ }^{111}$ In-DOTA-Cys-VS-PEG3400 antiCEA diabody (16). Possible explanations for the observed differences will be discussed later.

\section{Synthesis and Biodistribution of Monodispersed PEG27 AVP04-07}

Although conjugation of AVP04-07 to a PEG3400 moiety achieved reduced kidney uptake, PEG3400 would have major drawbacks as a clinical product because of its inherent polydispersity and the consequent inability to manufacture products that are easy to characterize chemically. Several monodispersed PEG building blocks are commercially available that can be converted into heterobifunctional reagents using peptide synthesis methodology. Thus, we first synthesized a DOTA-PEG27-Cys-VS derivative as summarized in Supplemental Scheme 1. Second, we conjugated DOTA-PEG27-Cys-VS to surface lysines of the diabody at $\mathrm{pH} 9.0$ at a molar ratio of 50:1 (22). When the conjugate was characterized by IEF and sodium dodecylsulfate gel electrophoresis (Supplemental Fig. 2), the expected shifts in $\mathrm{pI}$ and molecular size were observed. The ${ }^{111}$ In (Fig. 2, green trace) and ${ }^{125}$ I radiolabeled products were purified on Superdex 75, the major peak collected, and biodistribution studies performed. Inspection of the chromatogram and comparison to non-PEGylated diabody reveal a shift in apparent molecular size.

Biodistribution results are shown in Figures 3A and 3B. Despite the lower molecular weight of the PEG27 derivative than of the PEG3400 derivative, nearly equivalent results were obtained (compare with Fig. 1C). At 24 h,

FIGURE 2. Size-exclusion chromatography of radiolabeled non-PEGylated AVP04-07 diabody and its PEG conjugates. All samples were radiolabeled with ${ }^{111} \mathrm{In}$ and run on Superdex 75 size-exclusion column (radio activity in arbitrary units). Black = DOTA-nonPEGylated diabody conjugate; red = DOTACys-VS-PEG3400 diabody conjugate; green = DOTA-PEG27-Cys-VS

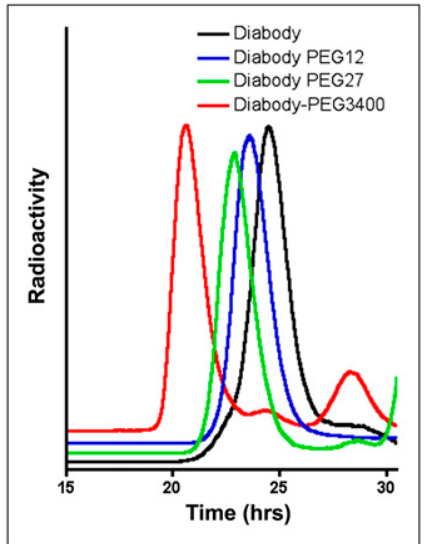
diabody conjugate; blue = DOTA-PEG12-Cys-VS diabody conjugate. 


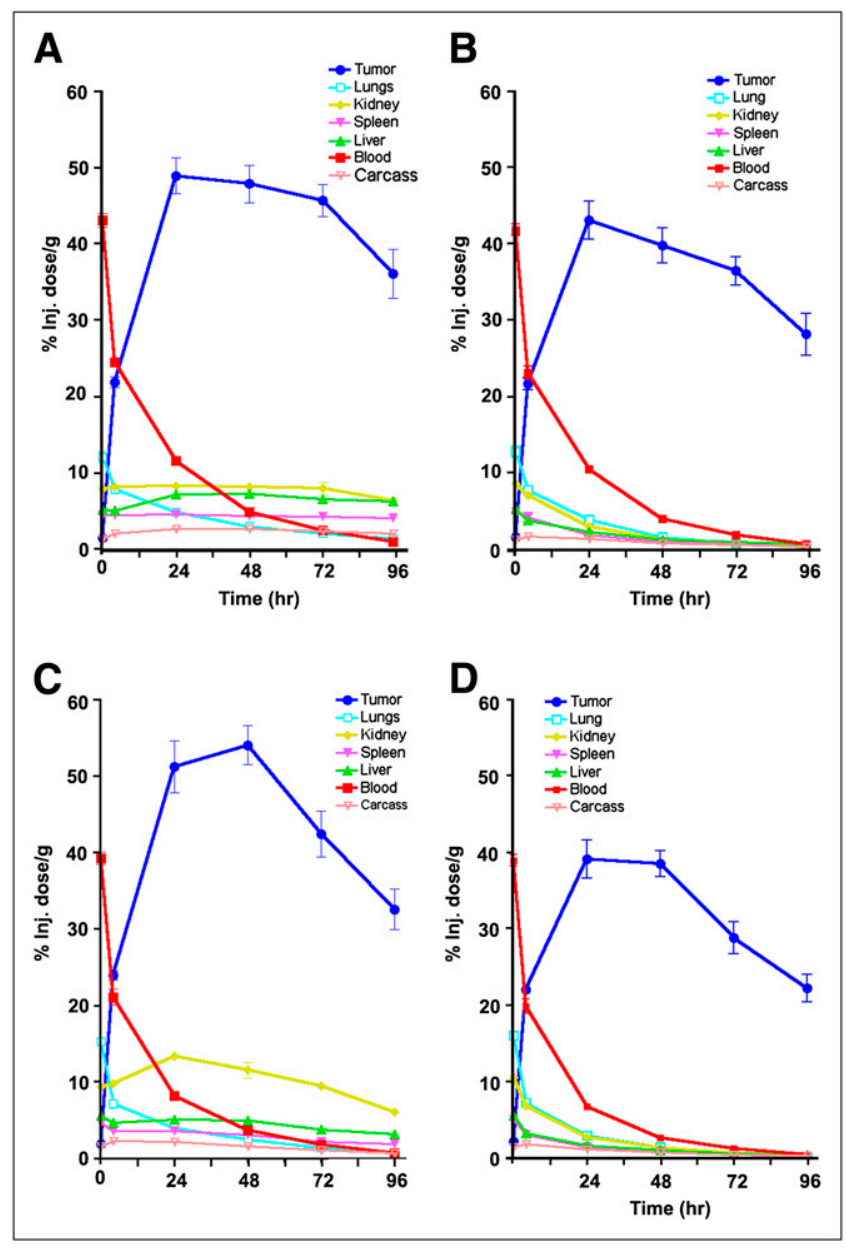

FIGURE 3. Biodistributions of DOTA-PEG27-Cys-VSAVP04-07 and DOTA-PEG12-Cys-VS-AVP04-07 measured in athymic mice bearing LS-174T xenografts: ${ }^{111}$ In-labeled PEG27 conjugate (A), ${ }^{125}$-labeled PEG27 conjugate (B), ${ }^{111}$ In-labeled PEG12 conjugate (C), and ${ }^{125}$-labeled PEG12 conjugate (D).

kidney uptake of ${ }^{111} \mathrm{In}$-labeled conjugate was $8.3 \% \mathrm{ID} / \mathrm{g}$, tumor uptake $49 \% \mathrm{ID} / \mathrm{g}$, and the tumor-to-blood ratio 4.2:1. Given the encouraging reduction in kidney uptake for the PEG27 derivative, we decided to synthesize and test the analogous PEG12 derivative.

\section{Synthesis and Biodistribution of Monodispersed PEG12 AVP04-07}

We synthesized DOTA-PEG12-Cys-VS (Supplemental Scheme 1) and conjugated it to AVP04-07 at pH 9.0 using molar ratios of 20:1 and 50:1. Unmodified AVP04-07 and the 2 conjugates were analyzed by high-resolution nanospray mass spectrometry to determine their degrees of substitution (Figs. 4A and 4C). The unmodified diabody gave a series of $\mathrm{m} / \mathrm{z}$ species that, when deconvoluted, had a calculated mass of 26,869 (Fig. 4A), in good agreement with that predicted by the amino acid sequence. For conjugation to DOTA-PEG12-Cys-VS at a molar ratio of
20:1, the deconvoluted peaks all differ from each other by multiples of 1,225 mass units (Fig. 4B), as expected for conjugation with DOTA-PEG12-Cys-VS. The mass differences are the same for the 50:1 molar ratio conjugation but are shifted to a higher degree of substitution (Fig. 4C). Using peak heights as an estimate, we calculate an average of 1.7 PEGs per monomer for the 20:1 conjugate and an average of 3.0 PEGs for the 50:1 conjugate. When each conjugate was test labeled with ${ }^{111} \mathrm{In}(178 \mathrm{mBq} / \mathrm{mg})$, the $20: 1$ conjugate gave $8.3 \%$ incorporation of radiolabel and the $50: 1$ gave $92 \%$ incorporation.

Biodistribution data for ${ }^{111} \mathrm{In}$ - and ${ }^{125}$ I-labeled DOTAPEG12-Cys-VS-AVP04-07 were similar to those for the PEG3400 and PEG27 derivatives (Figs. 3C and 3D). Notably, kidney uptake was initially higher $(13.4 \% \mathrm{ID} / \mathrm{g})$ for the PEG12 than the PEG27 conjugate but fell to lower levels $(6.1 \% \mathrm{ID} / \mathrm{g})$ by $96 \mathrm{~h}$, whereas tumor uptake and blood clearance are nearly identical for PEG12 and PEG27 conjugates.

\section{${ }^{64} \mathrm{Cu}$ PET of DOTA-PEG Conjugates of AVP04-07}

PET of ${ }^{64} \mathrm{Cu}$-labeled AVP04-07 (Fig. 5A) demonstrated relatively modest tumor uptake and high kidney uptake over $2 \mathrm{~d}$ for the non-PEGylated diabody. In contrast, the PEG12 and PEG27 conjugates showed relatively little kidney uptake throughout and high tumor uptake as early as 21-22 h (Figs. 5B and 5C). The measured tumor uptake for PEG12 was $49 \pm 3 \% \mathrm{ID} / \mathrm{g}$ at $46 \mathrm{~h}$, with a tumorto-blood ratio of $(9 \pm 4): 1$. These results are similar to those for the ${ }^{111}$ In-labeled conjugate and suggest that choice of radiometal has little effect on the biodistribution, an observation we made previously for an anti-CEA diabody (16).

\section{Comparative Imaging Characteristics of ${ }^{64} \mathrm{Cu}$ - and \\ ${ }^{111}$ In-Labeled AVP04-07 and Its DOTA-PEG Conjugates}

To compare different PEGylated versions of AVP04-07 radiolabeled with either ${ }^{111} \mathrm{In}$ or ${ }^{64} \mathrm{Cu}$, we examined 2 parameters: absolute tumor uptake and tumor-to-nontumor ratios using an IFOM (23). The biodistribution data were also replotted on the same graph for comparisons (Supplemental Fig. 3). The PEGylated versions outperform the non-PEGylated diabody in terms of both absolute tumor uptake and tumor-to-blood ratios for both ${ }^{111}$ In and ${ }^{64} \mathrm{Cu}$. The IFOM plots presented in Supplemental Figure 4 predict best imaging with either the ${ }^{64} \mathrm{Cu}$-labeled non-PEGylated diabody at $10 \mathrm{~h}$ or the ${ }^{64} \mathrm{Cu}$-labeled PEG12 version at $20 \mathrm{~h}$. With ${ }^{111}$ In, imaging is optimal with the PEG12 conjugate at $30-40 \mathrm{~h}$. However, when the background tissue is kidney, non-PEGylated diabody shows negative contrast until $50 \mathrm{~h}$ because of high renal uptake and retention of radiolabel. All PEGylated diabodies had similar image quality through $50 \mathrm{~h}$, with a slight edge to the PEG27 conjugate when imaged at $10-15 \mathrm{~h}$ for ${ }^{64} \mathrm{Cu}$ and $20-30 \mathrm{~h}$ for ${ }^{111} \mathrm{In}$.

\section{DISCUSSION}

The search for an effective radiolabeled antibody-based imaging agent has focused on antibody fragments for their 


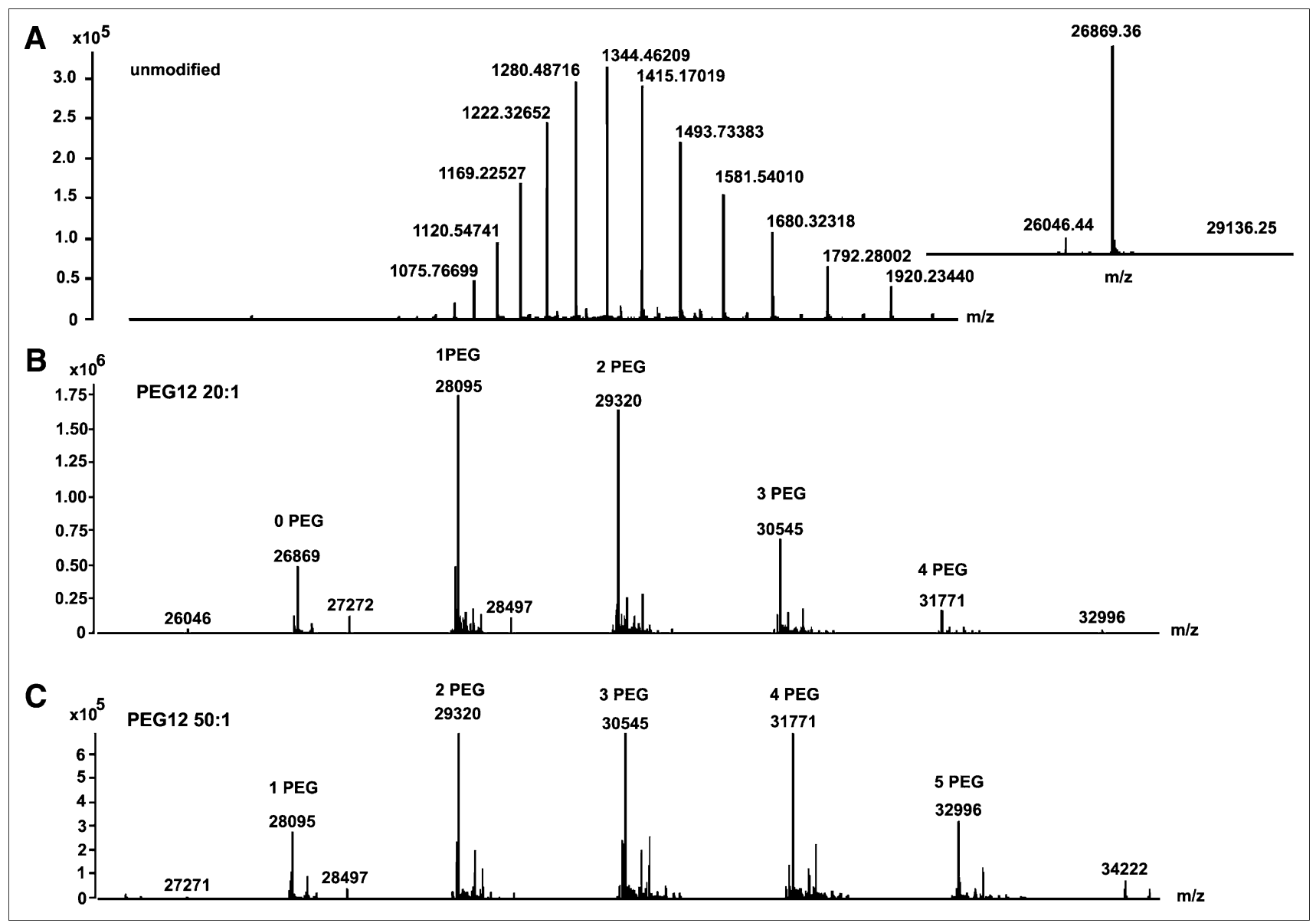

FIGURE 4. High-resolution nanospray mass spectrometry analysis of PEG12 AVP04-07 conjugates: raw mass spectrum for non-PEGylated diabody (inset: deconvoluted spectrum) (A), deconvolved spectrum for PEG12 conjugate $20: 1$ (B), and deconvolved spectrum for PEG12 conjugate 50:1 (C).

high tumor-to-blood ratios and on PET for its high sensitivity and quantitative results. We chose the diabody construct because it is the smallest bivalent recombinant antibody that can be produced directly in large quantities (24). However, if renal clearance of diabody is too rapid, the absolute tumor uptake will be low. In the case of radiometals such as ${ }^{64} \mathrm{Cu}$, for which tumor retention is greater than for radioiodine labels, its advantage is offset by prolonged kidney retention. Use of ${ }^{124} \mathrm{I}$ (5) avoids the kidney uptake issue due to metabolism and redistribution but at the expense of lower tumor uptake.

In this study our goal was to optimize the clearance parameters for ${ }^{64} \mathrm{Cu}$, which has an appropriate half-life (12 h), comparable to that of the diabody itself. PEGylation of diabody was selected because it has biodistributions similar to whole $\operatorname{IgG}$ or $\mathrm{F}\left(\mathrm{ab}^{\prime}\right) 2$ fragments but has the advantages of being engineered from a single gene rather than 2 and of not needing to be treated with a protease to reduce its molecular size. We previously approached this problem by conjugating a DOTA-PEG3400 derivative to an anti-CEA diabody, and although kidney uptake was reduced from 200 to $50 \% \mathrm{ID} / \mathrm{g}$ at $24 \mathrm{~h}$, we were unable to lower it to the levels (10-12\%ID/g at 24-48 h) seen with intact antiCEA IgG $(21,25)$. Factors that could explain the remaining high kidney uptake included the pI of the diabody $(17,18)$, the effect of binding to circulating antigen, or some unforeseen result of the conjugation chemistry. To gain insight into the problem, we selected a different diabody that, like anti-CEA diabody, shows strong tumor targeting in athymic mice bearing LS-174T xenografts. The antiTAG-72 antibody CC49 and a diabody derivative of CC49 have been previously described (26-29), and CC49 intact $\operatorname{IgG}$ has been used in many clinical studies $(25,30,31)$, making CC49 a good choice for a comparative study. Using a hexahistidine-tagged, E. coli-produced version of the CC49 diabody, namely AVP04-07, we found an absolute tumor uptake in the LS-174T mouse model at $24 \mathrm{~h}$ of 25 $\% \mathrm{ID} / \mathrm{g}$ (Fig. 1A), superior to that previously reported for an $(\mathrm{scFv})_{2}$ version of CC49 (4\% $\left.\mathrm{ID} / \mathrm{g}\right)(32)$.

For purposes of comparison, our first PEG construct (DOTA-Cys-VS-PEG3400) was identical to the one used with anti-CEA diabody (16). DOTA-Cys-VS-PEG3400 is a linear, bifunctional, polydispersed PEG that attaches to amino groups of diabodies via an active ester at one end 


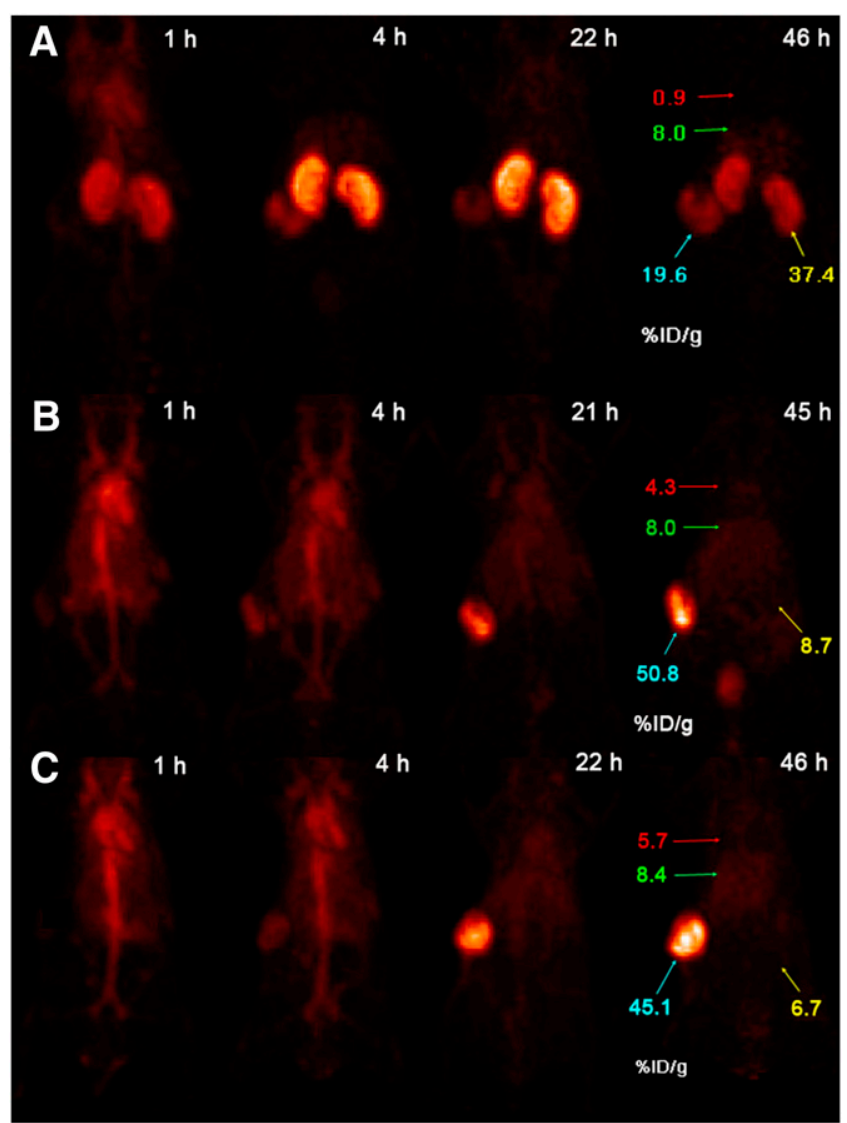

FIGURE 5. Serial PET of ${ }^{64} \mathrm{Cu}$ AVP04-07 and PEGylated AVP04-07 in athymic mice peripherally xenografted with TAG-72-expressing LS-174T colon tumors. Images are PET anterior-view maximum intensity projections normalized to reflect radiodecay-corrected relative image intensity per unit injected activity. Labels in red, green, yellow, and turquoise, respectively, show percentage injected dose (\%ID)/g in blood (heart), liver, kidney, and tumor as measured by direct assay after final scan: ${ }^{64} \mathrm{Cu}-\mathrm{AVP} 04-07$ (A), ${ }^{64} \mathrm{Cu}-\mathrm{PEG} 12$ AVP04-07 (B), and ${ }^{64} \mathrm{Cu}-\mathrm{PEG} 27$ AVP04-07 (C).

and to DOTA-Cys via vinyl sulfone at the other end. In contrast to anti-CEA diabody, kidney uptake was significantly lower $(10-12 \% \mathrm{ID} / \mathrm{g})$ than normally seen for intact IgGs. It is unlikely that the reduction in kidney uptake was due either to the conjugation chemistry or to the nature of the PEG agent (identical in both the anti-CEA and the antiCC49 cases); however, the reduction in kidney uptake could be due to either the final pI of the DOTA-PEG diabody conjugate or binding to circulating antigen. Regarding the $\mathrm{pI}$, both antibodies have a net negative charge after conjugation. Although negatively charged proteins are cleared less rapidly by the kidney than are positively charged proteins (18), the difference in pIs between the anti-CEA and anti-CC49 diabodies seems insufficient to account for their rather large differences in kidney uptake. Likewise, the role of circulating antigen in the kidney seems an unlikely explanation. Although it is well known that CEA is cleared rapidly by Kupffer cells in the liver (33), thus explaining the accumu- lation of ${ }^{111}$ In-DOTA-anti-CEA IgG in liver $(34,35)$, there is no obvious connection to the kidney. Although less is known about clearance of TAG-72, this high-molecular-weight acidic mucin is, like CEA, released into circulation, and its binding to diabody may be expected to protect the diabody from kidney filtration. The main argument against this idea is that only the PEGylated diabody has reduced kidney accumulation. Thus, further studies will have to be performed to determine the mechanisms causing the observed differences between the PEGylated anti-CEA and PEGylated anti-TAG-72 diabodies.

Although PEGylation is an attractive method for reducing kidney accumulation, oversubstitution may inhibit immunoreactivity $(10-12,36)$. In the case of anti-CEA diabody, it was necessary to limit the number of PEG3400s to about one per monomer (16). This fact, plus the polydispersed nature of PEG3400, prompted us to evaluate the effect of smaller DOTA-PEGs on kidney uptake. In the case of DOTA-PEG12-Cys-VS diabody, the degree of substitution was 3 PEG moieties per monomer as determined by high-resolution nanospray mass spectrometry. Notably, the biodistribution results for ${ }^{111}$ In-labeled PEG12 conjugate were similar to both the PEG3400 and the PEG27 conjugates, indicating that even PEGs of modest molecular size are efficient in reducing kidney uptake.

Based on the satisfactory biodistribution results with ${ }^{111} \mathrm{In}$, we went on to demonstrate high-quality PET of tumors both with ${ }^{64} \mathrm{Cu}$-labeled PEG12 and with ${ }^{64} \mathrm{Cu}$-labeled PEG27 conjugates by $24 \mathrm{~h}$, a time point that is well matched with the half-lives of both blood clearance and radionuclide.

All of the DOTA-PEG diabody conjugates had dramatically reduced kidney uptake, compared with non-PEGylated diabody, and were similar among themselves with regard to biodistribution (Fig. 1 and Supplemental Fig. 3). To further refine the comparison among the derivatives, we used a previously developed IFOM $(4,16,23,37)$. The analysis indicates modest advantages for the PEG12 conjugate when tumor-to-blood ratio is of primary concern and for the PEG27 conjugate when tumor-to-kidney contrast is the chief criterion.

\section{CONCLUSION}

On the basis of their excellent tumor and minimal kidney uptakes, we predict that monodispersed DOTA-PEGylated diabody conjugates will be suitable for human imaging of TAG-72-positive tumors. Further, we suggest that PEGylated diabodies may be an ideal platform for delivering other conjugated payloads to tumor.

\section{ACKNOWLEDGMENTS}

This research was supported by a research grant from Avipep Pty. Ltd., Parkville, Victoria, Australia. Avibody proteins were prepared and shipped by Harjit Khangura of Avipep. ${ }^{64} \mathrm{CuCl}_{2}$ was obtained from Washington University, St. Louis, MO, under NIH grant R24CA86307. 


\section{REFERENCES}

1. Wu AM, Olafsen T. Antibodies for molecular imaging of cancer. Cancer J. 2008; 14:191-197.

2. Williams LE, Wu AM, Yazaki PJ, et al. Numerical selection of optimal tumor imaging agents with application to engineered antibodies. Cancer Biother Radiopharm. 2001;16:25-35.

3. Holliger P, Hudson PJ. Engineered antibody fragments and the rise of single domains. Nat Biotechnol. 2005;23:1126-1136.

4. Williams LE, Lopatin G, Kaplan DD, Liu A, Wong JY. Update on selection of optimal radiopharmaceuticals for clinical trials. Cancer Biother Radiopharm. December 27, 2008 [Epub ahead of print].

5. Sundaresan G, Yazaki PJ, Shively JE, et al. ${ }^{124}$ I-labeled engineered anti-CEA minibodies and diabodies allow high-contrast, antigen-specific small-animal PET imaging of xenografts in athymic mice. J Nucl Med. 2003;44:1962-1969.

6. Tsai SW, Li L, Williams LE, Anderson A-L, Raubitschek AA, Shively JE. Metabolism and renal clearance of ${ }^{111}$ In-labeled DOTA conjugated antibody fragments. Bioconjug Chem. 2001;12:264-270.

7. Olafsen T, Kenanova VE, Sundaresan G, et al. Optimizing radiolabeled engineered anti-p185HER2 antibody fragments for in vivo imaging. Cancer Res. 2005;65:5907-5916.

8. Behr TM, Goldenberg DM, Becker W. Reducing the renal uptake of radiolabeled antibody fragments and peptides for diagnosis and therapy: present status, future prospects and limitations. Eur J Nucl Med. 1998;25:201-212.

9. Li L, Olafsen T, Anderson AL, Wu A, Raubitschek AA, Shively JE. Reduction of kidney uptake in radiometal labeled peptide linkers conjugated to recombinant antibody fragments: site-specific conjugation of DOTA-peptides to a Cys-diabody. Bioconjug Chem. 2002;13:985-995.

10. Yang K, Basu A, Wang M, et al. Tailoring structure-function and pharmacokinetic properties of single-chain Fv proteins by site-specific PEGylation. Protein Eng. 2003;16:761-770.

11. Delgado C, Pedley RB, Herraez A, et al. Enhanced tumour specificity of an anticarcinoembrionic antigen Fab' fragment by poly(ethylene glycol) (PEG) modification. Br J Cancer. 1996;73:175-182.

12. Lee LS, Conover C, Shi C, Whitlow M, Filpula D. Prolonged circulating lives of single-chain Fv proteins conjugated with polyethylene glycol: a comparison of conjugation chemistries and compounds. Bioconjug Chem. 1999;10:973-981.

13. Albrecht H, Denardo GL, Denardo SJ. Development of anti-MUC1 di-scFvs for molecular targeting of epithelial cancers, such as breast and prostate cancers. $Q J$ Nucl Med Mol Imaging. 2007;51:304-313.

14. Stork R, Campigna E, Robert B, Muller D, Kontermann RE. Biodistribution of a bispecific single-chain diabody and its half-life extended derivatives. $J$ Biol Chem. 2009;284:25612-25619.

15. Leong SR, DeForge L, Presta L, et al. Adapting pharmacokinetic properties of a humanized anti-interleukin- 8 antibody for therapeutic applications using sitespecific pegylation. Cytokine. 2001;16:106-119.

16. Li L, Yazaki PJ, Anderson $\mathrm{AL}$, et al. Improved biodistribution and radioimmunoimaging with poly(ethylene glycol)-DOTA-conjugated anti-CEA diabody. Bioconjug Chem. 2006;17:68-76.

17. Kobayashi H, Le N, Kim IS, et al. The pharmacokinetic characteristics of glycolated humanized anti-Tac Fabs are determined by their isoelectric points. Cancer Res. 1999;59:422-430.

18. Kim I, Kobayashi H, Yoo TM, et al. Lowering of pI by acylation improves the renal uptake of ${ }^{99 \mathrm{~m} T c-l a b e l e d}$ anti-Tac dsFv: effect of different acylating reagents. Nucl Med Biol. 2002;29:795-801.

19. Li L, Bading J, Yazaki PJ, et al. A versatile bifunctional chelate for radiolabeling humanized anti-CEA antibody with In-111 and Cu-64 at either thiol or amino groups: PET imaging of CEA-positive tumors with whole antibodies. Bioconjug Chem. 2008;19:89-96.

20. Lewis MR, Shively JE. Maleimidocysteineamido-DOTA derivatives: new reagents for radiometal chelate conjugation to antibody sulfhydryl groups undergo pH-dependent cleavage reactions. Bioconjug Chem. 1998;9:72-86.

21. Yazaki PJ, Wu AM, Tsai SW, et al. Tumor targeting of radiometal labeled antiCEA recombinant T84.66 diabody and $\mathrm{t} 84.66$ minibody: comparison to radioiodinated fragments. Bioconjug Chem. 2001;12:220-228.

22. Li L, Tsai SW, Anderson AL, Keire DA, Raubitschek AA, Shively JE. Vinyl sulfone bifunctional derivatives of DOTA allow sulfhydryl- or amino-directed coupling to antibodies: conjugates retain immunoreactivity and have similar biodistributions. Bioconjug Chem. 2002;13:110-115.

23. Williams LE, Liu A, Wu AM, et al. Figures of merit (FOMs) for imaging and therapy using monoclonal antibodies. Med Phys. 1995;22:2025-2027.

24. Yazaki PJ, Shively L, Clark C, et al. Mammalian expression and hollow fiber bioreactor production of recombinant anti-CEA diabody and minibody for clinical applications. J Immunol Methods. 2001;253:195-208.

25. Alvarez RD, Huh WK, Khazaeli MB, et al. A phase I study of combined modality ${ }^{90}$ yttrium-CC49 intraperitoneal radioimmunotherapy for ovarian cancer. Clin Cancer Res. 2002;8:2806-2811.

26. Santos AD, Kashmiri SV, Hand PH, Schlom J, Padlan EA. Generation and characterization of a single gene-encoded single-chain-tetravalent antitumor antibody. Clin Cancer Res. 1999;5(10, suppl)3118s-3123s.

27. Chauhan SC, Jain M, Moore ED, et al. Pharmacokinetics and biodistribution of ${ }^{177} \mathrm{Lu}$-labeled multivalent single-chain Fv construct of the pancarcinoma monoclonal antibody CC49. Eur J Nucl Med Mol Imaging. 2005;32:264-273.

28. Wittel UA, Jain M, Goel A, Chauhan SC, Colcher D, Batra SK. The in vivo characteristics of genetically engineered divalent and tetravalent single-chain antibody constructs. Nucl Med Biol. 2005;32:157-164.

29. Goel A, Baranowska-Kortylewicz J, Hinrichs SH, et al. ${ }^{99 \mathrm{~m}} \mathrm{Tc}-$ labeled divalent and tetravalent CC49 single-chain Fv's: novel imaging agents for rapid in vivo localization of human colon carcinoma. J Nucl Med. 2001;42:1519-1527.

30. Tempero M, Leichner P, Baranowska-Kortylewicz J, et al. High-dose therapy with ${ }^{90}$ yttrium-labeled monoclonal antibody CC49: a phase I trial. Clin Cancer Res. 2000;6:3095-3102.

31. Meredith RF, Bueschen AJ, Khazaeli MB, et al. Treatment of metastatic prostate carcinoma with radiolabeled antibody CC49. J Nucl Med. 1994;35: 1017-1022.

32. Beresford GW, Pavlinkova G, Booth BJ, Batra SK, Colcher D. Binding characteristics and tumor targeting of a covalently linked divalent CC49 singlechain antibody. Int J Cancer. 1999;81:911-917.

33. Gangopadhyay A, Bajenova O, Kelly TM, Thomas P. Carcinoembryonic antigen induces cytokine expression in Kupffer cells: implications for hepatic metastasis from colorectal cancer. Cancer Res. 1996;56:4805-4810.

34. Gangopadhyay A, Thomas P. Processing of carcinoembryonic antigen by Kupffer cells: recognition of a penta-peptide sequence. Arch Biochem Biophys. 1996;334:151-157.

35. Jessup JM, Petrick AT, Toth CA, et al. Carcinoembryonic antigen: enhancement of liver colonisation through retention of human colorectal carcinoma cells. $\mathrm{Br} \mathrm{J}$ Cancer. 1993;67:464-470.

36. Natarajan A, Xiong CY, Albrecht H, DeNardo GL, DeNardo SJ. Characterization of site-specific ScFv PEGylation for tumor-targeting pharmaceuticals. Bioconjug Chem. 2005;16:113-121.

37. Cai W, Olafsen T, Zhang X, et al. PET imaging of colorectal cancer in xenograftbearing mice by use of an ${ }^{18} \mathrm{~F}$-labeled $\mathrm{T} 84.66$ anti-carcinoembryonic antigen diabody. J Nucl Med. 2007;48:304-310. 